\title{
Metallicity of low-mass stars in Orion ${ }^{\star}$
}

 \\ 1 Dipartimento di Astronomia e Scienza dello Spazio, Università di Firenze, Largo E. Fermi 2, 50125 Firenze, Italy \\ 2 INAF - Osservatorio Astrofisico di Arcetri, Largo E. Fermi 5, 50125 Firenze, Italy \\ e-mail: vdorazi@arcetri.astro.it \\ 3 INAF - Osservatorio Astronomico di Palermo, Piazza del Parlamento 1, 90134 Palermo, Italy
}

Received 28 October 2008 / Accepted 18 April 2009

\begin{abstract}
Context. Determining the metal content of low-mass members of young associations provides a tool that addresses different issues, such as triggered star formation or the link between the metal-rich nature of planet-host stars and the early phases of planet formation. The Orion complex is a well known example of possible triggered star formation and is known to host a rich variety of proto-planetary disks around its low-mass stars. Available metallicity measurements yield discrepant results.

Aims. We analyzed FLAMES/UVES and Giraffe spectra of low-mass members of three groups/clusters belonging to the Orion association. Our goal is the homogeneous determination of the metallicity of the sample stars, which allows us to look for [Fe/H] differences between the three regions and for the possible presence of metal-rich stars.

Methods. Nine members of the ONC and one star each in the $\lambda$ Ori cluster and OB1b subgroup were analyzed. After the veiling determination, we retrieved the metallicity by means of equivalent widths and/or spectral synthesis using MOOG.

Results. We obtain an average metallicity for the $\mathrm{ONC}[\mathrm{Fe} / \mathrm{H}]=-0.01 \pm 0.04$. No metal-rich stars were detected and the dispersion within our sample is consistent with measurement uncertainties. The metallicity of the $\lambda$ Ori member is also solar, while the OB $1 \mathrm{~b}$ star has an $[\mathrm{Fe} / \mathrm{H}]$ significantly below the $\mathrm{ONC}$ average. If confirmed by additional $[\mathrm{Fe} / \mathrm{H}]$ determinations in the OB $1 \mathrm{~b}$ subgroup, this result would support the triggered star formation and the self-enrichment scenario for the Orion complex.
\end{abstract}

Key words. stars: pre-main sequence - stars: abundances - open clusters and associations: individual: Orion - stars: planetary systems

\section{Introduction}

The Orion OB1 association, located at roughly $400 \mathrm{pc}$, is probably the best known region of active star formation. It comprises four major subgroups of different ages, sizes, and positions $(1 \mathrm{a}, 1 \mathrm{~b}, 1 \mathrm{c}, 1 \mathrm{~d}$ where the Orion nebula cluster - ONC is), together with several grouping of stars and clusters, covering a wide range of ages and environmental conditions: extremely young groups of stars are present still embedded in their parental clouds, 3-5 Myr old clusters like $\sigma$ and $\lambda$ Ori, as well as older populations like the Ori Ia sub-association (age $\sim 10 \mathrm{Myr}$ ). Orion is since long considered as a possible example of triggered star formation (Blaauw 1991, ASI Ser. 342, p. 125), supported by the increasing ages between the Id/Ic, Ib, and Ia subgroups and by the different content of gas and dust of the different regions. In the triggered scenario, winds and $\mathrm{SN}$-driven shock waves originating from a first generation of massive stars induce the formation of a new group/cluster of stars. This second generation population is likely contaminated by the enriched ejecta of the massive stars of the first generation (e.g., Reeves 1978) and thus might show a different abundance pattern. The secure and homogeneous determination of the chemical composition across Orion, and the comparison of the abundance patterns of the different subgroups, hence represent a critical and independent test of this scenario.

* Based on observations collected at Paranal Observatory, ESO (Chile). Programs 072.D-0019, 074.C-0757, 076.D-0136, 076.C-0145.

$\star \star$ We dedicate this paper to the memory of Roberto Pallavicini who prematurely passed away during the completion of this work. We all miss him sorely.
Besides providing insights into triggered star formation models, the determination of the metallicity of low-mass stars in young associations is important in the context of extra solar planets. It is now well-established that planet-host stars are on average more metal-rich than stars without planetary-mass companions (Gonzalez 1997; Gonzalez et al. 2001; Santos et al. 2001; Fisher \& Valenti 2005). The mean metallicity of stars hosting a gas giant planet is $[\mathrm{Fe} / \mathrm{H}]=+0.15 \pm 0.23$, compared to the value $[\mathrm{Fe} / \mathrm{H}]=-0.1 \pm 0.18$ for the solar neighborhood. Also, the frequency of giant planets around metal-rich stars $([\mathrm{Fe} / \mathrm{H}] \geq 0.3)$ is $f_{\mathrm{p}} \geq 20 \%$, much higher than that of planets around solar (or under-solar) metallicity stars $\left(f_{\mathrm{p}} \sim 3 \%\right)$. More important, complementary studies suggest that the high metal content is primordial and not due to pollution by cannibalized planetary bodies (Ecuvillon et al. 2006; Gilli et al. 2006; Pinsonneault et al. 2001; see however Pasquini et al. 2007, for a different view).

Surveys of the properties of planet-host stars provide clues to the mechanism of planet formation based on its final product (i.e., the already formed planet), since the majority of field stars where a giant planet has been detected are older than $\sim 1$ Gyr. On the other hand, circumstellar disks around pre-main sequence (PMS) stars, which are the birth sites of planets, are ubiquitously found in star-forming regions (SFRs). The question then is what the metallicity of low-mass stars that are likely to be forming planets now in SFRs and young associations might be. To definitively answer it, the metallicity needs to be derived, as much as possible, for a complete SFR sample. The Orion complex already represents an excellent target, given the high frequency and wide variety of proto-planetary disks around its low-mass members. 
In the last 50 years, hundreds of studies have investigated key aspects related to the Orion stellar population, as well as to the associated HII regions and the surrounding interstellar medium. However, considerably less attention has been given to determining its chemical composition. Available information on the abundance pattern in Orion and, in particular, of the ONC, mostly comes from studies of the ionized gas in the Orion nebula or from abundance determination in stars of early spectraltype. The latter, however, is affected by several uncertainties, for example, NLTE and rotational broadening of the spectral lines. Few measurements of the metallicity among lower-mass stars have so far been carried out, as we discus in Sect. 2 below. With this background in mind, we exploited the available high-resolution spectra acquired in the framework of two different projects (Palla et al. 2005, 2007; Sacco et al. 2008) to measure the metallicity in a sample of ONC members and in one star belonging to the $\lambda$ Ori cluster. One member of the Orion OB1b subgroup, whose spectrum was retrieved from the ESO archive, was also analyzed.

The paper is organized as follows. In Sect. 2 we give an overview of previous determination of metallicity in Orion members; in Sect. 3 we describe the sample, observations, and data reduction. The analysis is presented in Sect. 4 and the results and discussion in Sect. 5, followed by the conclusions (Sect. 6).

\section{Previous metallicity determinations in Orion}

Based on the determination of oxygen in the Orion nebula by Osterbrock et al. (1992), it has been believed until recently that the metallicity of Orion is lower than that of the Sun, representing a problem for Galactic evolution models in the solar vicinity. While the reappraisal of oxygen in the Orion nebula by the study of Esteban (2004) no longer supported this view, stellar abundances obtained by Cunha \& Lambert (1994) and Cunha et al. (1998) also suggested a lower-than-solar metallicity for the whole Orion complex. Specifically, Cunha and collaborators measured $\mathrm{Fe}, \mathrm{O}, \mathrm{C}, \mathrm{N}$, and $\mathrm{Si}$ abundances in a sample of $\mathrm{B}$ - and $\mathrm{F} / \mathrm{G}$ - type Orion members, primarily belonging to the subgroups $1 \mathrm{a}$ and $1 \mathrm{c}$. They found that the metallicity $([\mathrm{Fe} / \mathrm{H}])$ ranges between -0.31 (Ic, G-type star) and +0.14 (Ia, B-type star); in spite of this rather wide interval Cunha and collaborators concluded that all the four subgroups 1a, 1b, 1c, and 1d (where the ONC is located) are characterized by the same, somewhat below solar, metallicity $([\mathrm{Fe} / \mathrm{H}]=-0.08 \pm 0.13$, where the error is the $1 \sigma$ deviation from the mean). $\mathrm{F}$ and $\mathrm{G}$-type stars only would indicate an even lower metal content $([\mathrm{Fe} / \mathrm{H}]=-0.13 \pm 0.13)$. Interestingly, the most metal-rich among the $\mathrm{F} / \mathrm{G}$-type stars is the only ONC members included in the sample. For this star, $\mathrm{P} 1455$, they derived a metallicity $[\mathrm{Fe} / \mathrm{H}]=+0.08 \pm 0.15$. At variance with their claim of a homogeneous iron content, Cunha and collaborators $(1992,1998)$ found evidence of star-to-star variations in $\mathrm{O}$ and $\mathrm{Si}$ abundances, with a few $\mathrm{O}$ - and Si-enhanced stars being more centrally located (in the Trapezium region) than the O- and Si-poor ones, distributed throughout the association. The authors suggest that the observed abundance pattern could be evidence of the expected self-enrichment. More recently, Simón-Díaz et al. (2006) derived oxygen abundances for a few B-type stars in the Trapezium finding lower values than those of Cunha \& Lambert and, in contrast to their results, no evidence of any elemental enhancement.

Focusing on low-mass stars, seven objects in Orion 1c and 1d were included in the study of Padgett (1996), who derived [Fe/H] values in the interval -0.1 to $+0.23 \mathrm{dex}$ and an average $[\mathrm{Fe} / \mathrm{H}]$ slightly above solar. Of the four ONC stars included in the sample, two showed slightly over-solar abundances $([\mathrm{Fe} / \mathrm{H}]=$ $0.14 \pm 0.18$ and $0.08 \pm 0.17$ respectively), and the other two stars a solar metallicity $([\mathrm{Fe} / \mathrm{H}]=-0.01 \pm 0.21,-0.01 \pm 0.17)$. Finally, based on three stars, Santos et al. (2008) obtained an average for the $\mathrm{ONC}[\mathrm{Fe} / \mathrm{H}]=-0.13 \pm 0.06$, again below solar. In summary, existing determinations of the metal content in the whole Orion complex reach controversial conclusions both on the overall metallicity - in particular on whether it is below solar or not on the presence of a star-to-star scatter within a given subgroup (the $\mathrm{ONC}$ in particular), and on differences in $[\mathrm{Fe} / \mathrm{H}]$ between the different subgroups.

\section{Sample stars and observations}

\subsection{ONC}

The spectra were acquired in two observing sessions with FLAMES on VLT/UT2 (Pasquini et al. 2000) as part of a project focused on measurements of lithium abundances in very lowmass members of the ONC (Palla et al. 2005, 2007). In both runs Giraffe fibers were mostly allocated to M-type stars; instead, UVES fibers were used to observe K- and M-type stars with the goal of deriving their accretion rates by means of the Ca II infrared triplet (Flaccomio et al., in prep.). The present sample includes all the K-type stars observed with UVES (seven stars), while $\mathrm{M}$ dwarfs were discarded since determination of metallicity in these cool stars is not feasible with standard methods/codes and Kurucz model atmospheres (see Sect. 4.3.1 below). To the UVES sample, we added the only two K-type stars observed with Giraffe and for which Palla et al. (2005) derived a negligible veiling. The total sample hence consists of nine stars.

The observations were carried out on 15 February (Visitor Mode) and 15 November (Service Mode), 2004. The UVES spectrograph (Dekker et al. 2000) in fiber mode provides a resolution $R=40000$. We used the CD4 cross-disperser (needed to target the Ca II triplet), allowing us to cover a spectral range from $\sim 6700 \AA$ to $10000 \AA$. Giraffe was used with the HR15 setup, yielding a resolution $R \sim 19000$ and spectral coverage from 660.7 to $696.5 \mathrm{~nm}$.

The sample stars are listed in Table 1, where we give the identifier from Hillenbrand (1997), an observing log (the dates of the observations, total exposure time, $S / N$ ratios), along with the stellar properties ( $I$ magnitude, $V-I$ color, spectral-type, rotational period, effective temperature $-T_{\text {eff }}-$, and $K$-band excess) are also listed in the table. Information on photometry, spectral-types, effective temperatures, and excess were taken from Hillenbrand (1997) and Hillenbrand et al. (1998), while rotational periods come from Herbst et al. (2002).

Reduction of UVES data was carried out using the FLAMES-UVES pipeline, following the standard procedure: bias subtraction, flat-field correction, order extraction and wavelength calibration. Sky subtraction was performed outside the pipeline context, using the fiber allocated to the sky. Spectra of the same star obtained in different exposures were then coadded, after checking that no radial velocity variations were present. Giraffe data were reduced using the BLDRS software (Blecha \& Simond 2004 - see details in Palla et al. 2007). Final $S / N$ ratios are in the range $70-125$ for the UVES spectra and 150-200 for the Giraffe ones. All UVES spectra are shown in Fig. 1.

\section{2. $\lambda$ Ori and Orion $O B 1 b$}

As in the case of ONC, we selected stars of $\lambda$ Ori from a wider sample acquired to investigate membership and accretion 
Table 1. ONC target stars, observing log, and target properties ${ }^{1}$.

\begin{tabular}{|c|c|c|c|c|c|c|c|c|c|c|c|}
\hline $\begin{array}{r}\text { Star } \\
(\mathrm{H} 97) \\
(1)\end{array}$ & 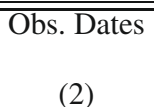 & Instrument & $\begin{array}{l}\text { Exp. time } \\
\text { (sec) } \\
(4)\end{array}$ & 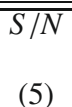 & $\begin{array}{l}I \\
(6)\end{array}$ & $\begin{array}{c}V-I \\
\text { (7) }\end{array}$ & Sp. Type & $\begin{array}{l}T_{\text {eff }} \\
(\mathrm{K}) \\
(9)\end{array}$ & $\begin{array}{c}\Delta(I-K) \\
(\mathrm{mag}) \\
(10)\end{array}$ & $\begin{array}{c}P \\
\text { (days) } \\
(11)\end{array}$ & $\begin{array}{c}v_{\text {rad }} \\
\left(\mathrm{km} \mathrm{s}^{-1}\right) \\
(12)\end{array}$ \\
\hline $223 a$ & $15-02-04$ & UVES, CD4 & $3 \times 3600$ & 70 & 13.64 & 2.42 & K5 & 4395 & 0.48 & - & $25.43 \pm 1.58$ \\
\hline 268 & $15-02-04$ & UVES, CD4 & $3 \times 3600$ & 95 & 12.88 & 1.61 & K5-K6 & 4197 & 0.16 & 9.81 & $27.80 \pm 1.02$ \\
\hline 278 & $15-02-04$ & UVES, CD4 & $3 \times 3600$ & 70 & 13.66 & 1.94 & $\mathrm{~K} 2-\mathrm{K} 7 \mathrm{e}$ & 4395 & 1.69 & 6.76 & $24.58 \pm 1.49$ \\
\hline 487 & $15-02-04$ & UVES, CD4 & $3 \times 3600$ & 105 & 12.79 & 1.78 & K6 & 4197 & 0.28 & - & $29.27 \pm 0.87$ \\
\hline 673 & $15-02-04$ & UVES, CD4 & $3 \times 3600$ & 110 & 13.07 & 1.83 & K5 & 4395 & 0.81 & 1.44 & $24.53 \pm 1.29$ \\
\hline 683 & $15-02-04$ & UVES, CD4 & $3 \times 3600$ & 130 & 11.76 & 1.85 & K6 & 4197 & 0.28 & 11.50 & $25.73 \pm 1.51$ \\
\hline 907 & $15-11-04$ & UVES, CD4 & $4 \times 2600$ & 125 & 12.77 & 1.60 & K1-K4 & 4775 & - & - & $20.82 \pm 1.24$ \\
\hline 664 & $15-02-04$ & GIRAFFE & $3 \times 3600$ & 150 & 14.05 & 2.88 & K5.5-K7 & 4197 & -0.18 & 7.20 & $25.36 \pm 1.18$ \\
\hline 1020 & $15-02-04$ & GIRAFFE & $3 \times 3600$ & 200 & 12.65 & 1.40 & K6 & 4197 & -0.01 & - & $27.60 \pm 1.82$ \\
\hline
\end{tabular}

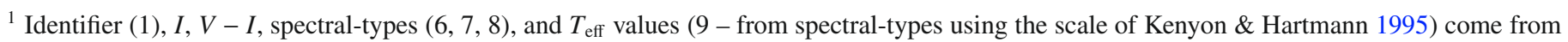
Hillenbrand (1997); $K$-band excess (10) and rotational periods (11) were taken from Hillenbrand et al. (1998) and Herbst et al. (2002), respectively. Radial velocities (12) were measured from our spectra.



Fig. 1. UVES spectra of the ONC sample stars in the wavelength range $6740-6780 \AA$. Different features are marked. Note the shallower lines in the spectrum of star h278, for which we derived a high veiling value. properties in this young cluster by Sacco et al. (2008). To select the stars, we applied the same criteria used for the ONC sample, discarding the fast rotating members $\left(v \sin i \geq 30 \mathrm{~km} \mathrm{~s}^{-1}\right.$ ), binaries, and stars with spectral-type later than $\mathrm{K} 7$; this criterion resulted in the selection of one star only (Dolan 24). The observations were carried out in Service Mode during October and November, 2005, using FLAMES-UVES and CD\#3 crossdisperser, covering the spectral interval 4840-6840 $\AA$. We enlarged our sample with a member of the OB1b association (HD 294297) whose FLAMES-UVES spectrum, obtained with the CD\#4 cross-disperser, was retrieved from the ESO Archive (Program 076.C-0145, PI Jeffries). This star is included in the sample of Cunha et al. (1998) and thus allows a direct comparison of their and our abundance scales. For both stars data reduction was performed under ESO-MIDAS context and using the FLAMES/UVES pipeline. The two targets along with information on their properties and observations are listed in Table 2; their spectra are shown in Fig. 2.

\section{Analysis and abundance determination}

\subsection{Radial velocities}

Radial velocities were computed from our spectra using the task RVIDLINES in IRAF ${ }^{2}$ and a list of 10 lines; with the exception of Dolan 24, all measurements are in good agreement with previous determinations in the literature (Sicilia-Aguilar et al. 2005; Caballero 2007). For Dolan 24 we instead obtain a radial velocity lower than the measurement of Dolan \& Mathieu (1999-29.14 $\mathrm{km} \mathrm{s}^{-1}$ ) and slightly below two different estimates of the average radial velocity of $\lambda$ Ori $\left(v_{\text {rad }}=24.3 \pm 2.8 \mathrm{~km} \mathrm{~s}^{-1}\right.$ - Dolan \& Mathieu 1999; $v_{\mathrm{rad}}=27.3 \pm 0.49 \mathrm{~km} \mathrm{~s}^{-1}-$ Sacco et al. 2008), suggesting that this star likely is a spectroscopic

\footnotetext{
${ }^{2}$ IRAF is distributed by the National Optical Astronomical Observatories, which are operated by the Association of Universities for Research in Astronomy, under contract with the National Science Foundation.
} 
Table 2. $\lambda$ Ori and OB1b targets, observing log, and target properties ${ }^{1}$.

\begin{tabular}{|c|c|c|c|c|c|c|c|c|c|}
\hline $\begin{array}{l}\text { Star } \\
\text { (1) }\end{array}$ & $\begin{array}{l}\text { Obs. Dates } \\
\text { (2) }\end{array}$ & $\begin{array}{c}\text { Instrument } \\
\text { (3) }\end{array}$ & $\begin{array}{c}\text { Exp. time } \\
\text { (s) } \\
\text { (4) }\end{array}$ & $\begin{array}{c}S / N \\
(5)\end{array}$ & $\begin{array}{l}I \\
\text { (6) }\end{array}$ & $\begin{array}{c}R-I \\
(7)\end{array}$ & $\begin{array}{c}\text { Sp. Type } \\
\text { (8) }\end{array}$ & $\begin{array}{l}T_{\text {eff }} \\
(\mathrm{K}) \\
(9)\end{array}$ & $\begin{array}{c}v_{\text {rad }} \\
\left(\mathrm{km} \mathrm{s}^{-1}\right) \\
(10)\end{array}$ \\
\hline HD 294297 & $2005-11-17$ & UVES, CD4 & $3 \times 3575$ & 150 & - & - & G0 & 6100 & $26.46 \pm 0.41$ \\
\hline Dolan24 & $\begin{array}{l}2005-10-16 / 17 / 18 \\
2005-11-11\end{array}$ & UVES, CD3 & $4 \times 2760$ & 200 & 11.75 & 0.53 & - & 5080 & $22.83 \pm 0.53$ \\
\hline
\end{tabular}

${ }^{1}$ Information for star HD 294297 comes from the SIMBAD database. Identifiers and photometry of Dolan 24 in $\lambda$ Ori were taken from Dolan \& Mathieu (1999). $T_{\text {eff }}$ for HD 294297 was retrieved from Cunha et al. (1998), while that of Dolan 24 was estimated from the $R-I$ color. Radial velocities were measured from our spectra.

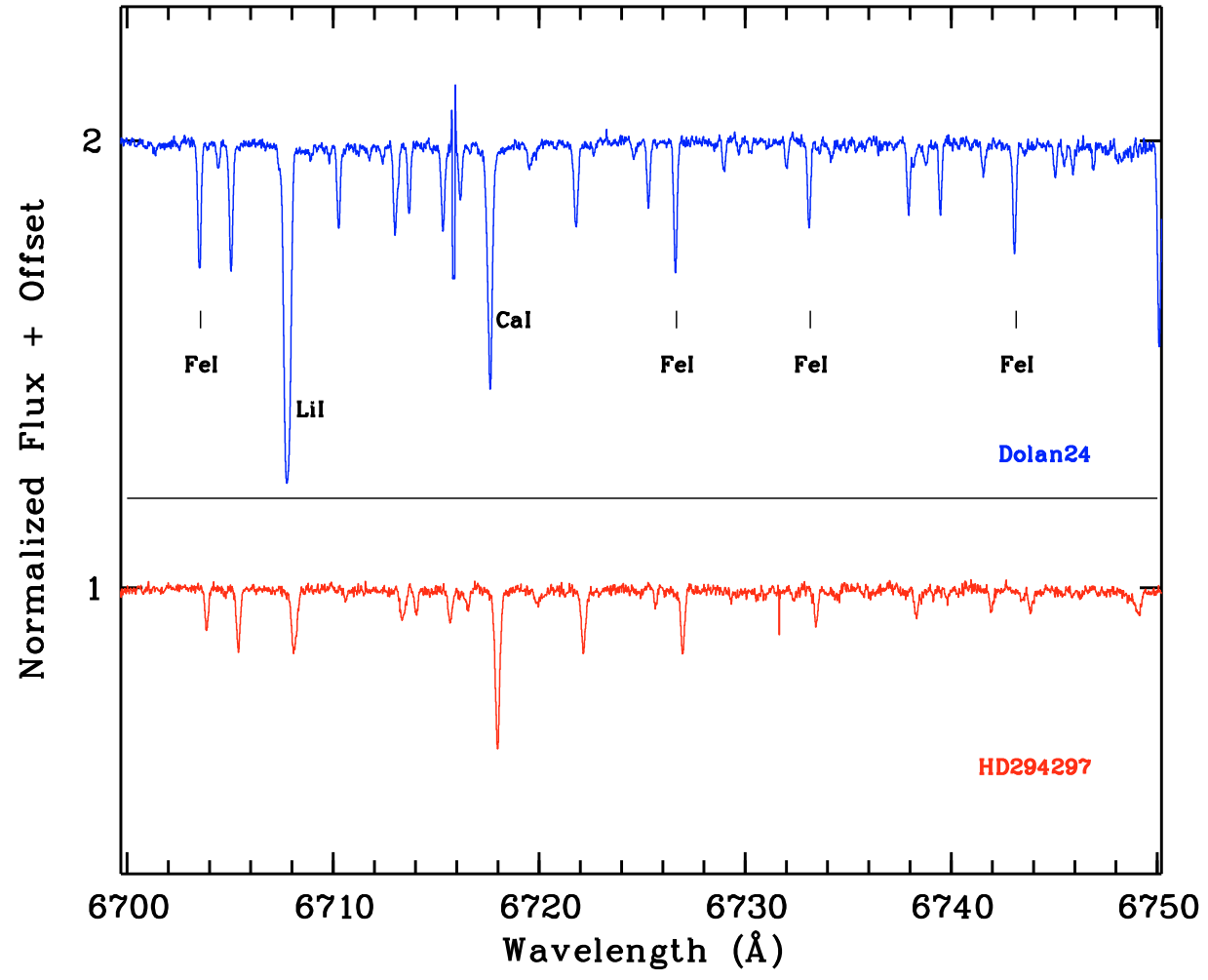

Fig. 2. Same as Fig. 1, but UVES spectra of the $\lambda$ Ori star (upper panel) and of the OB1b member are shown. Note the different spectral interval displayed here with respect to Fig. 1. binary. Derived radial velocities for all stars are listed in the last columns of Tables 1 and 2.

\subsection{Estimate of veiling}

As well known, spectra of PMS stars might be affected by spectral veiling that alters measured equivalent widths (EWs), since photospheric absorption lines are filled in by accretion shock emission. As a consequence, the observed lines are weaker than intrinsic ones and one needs to correct for veiling before deriving abundances. This is accomplished by determining $r$, the ratio of the excess to the photospheric continuum; then the relationship between the true and measured $E W \mathrm{~s}$ is $E W_{0}=E W_{\text {meas }} \times(1+r)$.

As mentioned in the previous section, veiling for the two ONC stars observed with Giraffe was already derived by Palla et al. (2005); both of them have $r \approx 0$, and this was indeed the reason why we included them in our sample. On the other hand, we directly estimated $r$ from our spectra for the seven ONC stars observed with UVES and for the $\lambda$ Ori member (HD 294297 should not be affected by veiling - Cunha et al. 1995), following a procedure similar to that described in Palla et al. (2005, 2007), but using different features. Namely, we measured the $E W \mathrm{~s}$ of selected strong lines in the target stars and compared them with those measured in the spectra of 11 members of the IC 2602 and IC 2391 clusters, which are old enough ( 30-50 Myr) to ensure that their spectra are not affected by veiling. The IC clusters stars cover the same range of effective temperatures as the Orion targets and their spectra are characterized by a similar resolution (see Stauffer et al. 1997; Randich et al. 2001). For the 580 setup we used nine lines; namely, Ca I 5857.5 А, Ca I $6102.7 \AA$, Ca I $6122.2 \AA$, Fe I $6546.3 \AA$, Ni I $6643.6 \AA$, Fe I 6662.5 AA, V I $6624.8 \AA$, Ca I $6717.7 \AA$, Ti I $6743.2 \AA$. For the 860 nm setup we employed six lines in the range $6743-8085 \AA$; namely, $6743.12 \AA$ (Fe I), 7148.15 $(\mathrm{Ca}$ I), $7445.75 \AA$ (Fe I), $7698.97 \AA$ (K I), $7937.13 \AA$ (Fe I), 7998.97 $\mathrm{A}$ (Fe I).

As a first step, we checked whether a dependence of the strength of the lines on temperature was present among IC cluster members: with the exception of the Ca I 6122.2 $\AA$ and K I $7698.97 \AA$ features, all the other lines did not show strong trends with $T_{\text {eff }}$ within $\sim 300 \mathrm{~K}$. Hence, in these cases we derived the mean $E W$ considering all stars in the two clusters and used this average value for comparison with the Orion stars. For the two features whose strength depends on effective temperature, we instead used the $E W$ value of the IC cluster star with $T_{\text {eff }}$ closest to 
Table 3. Final stellar parameters and metallicity values derived for ONC $\operatorname{stars}^{1}$.

\begin{tabular}{|c|c|c|c|c|c|c|c|}
\hline $\begin{array}{l}\text { Star } \\
\text { (1) }\end{array}$ & $\begin{array}{l}r \\
(2)\end{array}$ & $\begin{array}{l}T_{\text {eff }} \\
(\mathrm{K}) \\
(3)\end{array}$ & $\begin{array}{c}\log g \\
(4)\end{array}$ & $\begin{array}{c}\xi \bar{\xi} \\
\left(\mathrm{km} \mathrm{s}^{-1}\right) \\
(5)\end{array}$ & $\begin{array}{c}v \sin i \\
\left(\mathrm{~km} \mathrm{~s}^{-1}\right) \\
(6)\end{array}$ & $\begin{array}{c}{[\mathrm{Fe} / \mathrm{H}] \pm \sigma_{1}} \\
(E W \mathrm{~s}) \\
(7)\end{array}$ & $\begin{array}{l}\sigma_{2} \\
\quad \text { (Spec. Synth.) } \\
\\
\text { (8) }\end{array}$ \\
\hline $223 a$ & $0.02 \pm 0.04$ & 4450 & 3.8 & 1.6 & - & $0.03 \pm 0.06 \pm 0.07$ & - \\
\hline 268 & $0.03 \pm 0.04$ & 4300 & 3.9 & 1.6 & $10 \pm 3$ & $-0.08 \pm 0.16 \pm 0.09$ & $-0.1 \pm 0.1 \pm 0.1$ \\
\hline 278 & $0.8 \pm 0.07$ & 4395 & - & - & - & - & - \\
\hline 487 & $0.02 \pm 0.02$ & 4300 & 3.9 & 1.7 & $15 \pm 3$ & $-0.07 \pm 0.08 \pm 0.09$ & $0 \pm 0.1 \pm 0.09$ \\
\hline 673 & $0.04 \pm 0.03$ & 4700 & 4.0 & $1.5^{*}$ & $20 \pm 3$ & $0.0 \pm 0.1 \pm 0.07$ & $0 \pm 0.1 \pm 0.08$ \\
\hline 683 & $0.0 \pm 0.02$ & 4250 & 3.3 & 1.6 & $13 \pm 3$ & $0.01 \pm 0.1 \pm 0.08$ & $-0.1 \pm 0.1 \pm 0.09$ \\
\hline 907 & $0.01 \pm 0.03$ & 4775 & 4.1 & 1.5 & $35 \pm 3$ & - & $0.0 \pm 0.1 \pm 0.11$ \\
\hline 664 & $0^{* *}$ & 4200 & 4.1 & 1.6 & $13 \pm 3$ & - & $0.05 \pm 0.1 \pm 0.1$ \\
\hline 1020 & $0^{* *}$ & 4200 & 3.8 & 1.6 & $13 \pm 3$ & - & $0.0 \pm 0.1 \pm 0.1$ \\
\hline VXR76A (list CD3 ) & - & 4340 & 4.5 & 1.2 & $8 \pm 3$ & $0.00 \pm 0.06 \pm 0.09$ & $0 \pm 0.1 \pm 0.1$ \\
\hline VXR76A (list $\left.{ }_{\text {CD4 }}\right)$ & - & 4400 & 4.5 & 1.3 & $8 \pm 3$ & $-0.01 \pm 0.08 \pm 0.08$ & $0 \pm 0.1 \pm 0.1$ \\
\hline
\end{tabular}

* The initial microturbulence was adopted for this star, since $\xi$ could not be optimized due to the lack of lines covering a wide enough $E W$ interval. ** $r$ values for stars 664 and 1020 come from Palla et al. (2005).

${ }^{1}$ In the table we list the star ID, the veiling $r$, adopted stellar parameters, projected rotational velocities, and the final [Fe/H] values, from the $E W$ and synthesis analysis, along with $\sigma_{1}$ and $\sigma_{2}$ errors (see text). For $[\mathrm{Fe} / \mathrm{H}]$ from $E W \mathrm{~s}$ we also provide in parenthesis the number of employed $\mathrm{Fe} \mathrm{I}$ lines.

Table 4. Same as Table 3, but the results for $\lambda$ Ori targets and star HD 294297 are listed.

\begin{tabular}{|c|c|c|c|c|c|c|c|}
\hline $\begin{array}{l}\text { Star } \\
\text { (1) }\end{array}$ & $\begin{array}{l}r \\
(2)\end{array}$ & $\begin{array}{l}T_{\text {eff }} \\
(\mathrm{K}) \\
(3)\end{array}$ & $\begin{array}{c}\log g \\
(4)\end{array}$ & $\begin{array}{c}\xi \\
\left(\mathrm{km} \mathrm{s}^{-1}\right) \\
(5)\end{array}$ & $\begin{array}{c}v \sin i \\
\left(\mathrm{~km} \mathrm{~s}^{-1}\right) \\
(6)\end{array}$ & $\begin{array}{r}\overline{F e} / \mathrm{H}] \pm \sigma \\
(E W \mathrm{~s}) \\
(7)\end{array}$ & $\begin{array}{lr}\sigma_{2} & \\
& \text { (Spec. Synth.) } \\
& \\
& (8)\end{array}$ \\
\hline HD 294297 & - & 6100 & 4.0 & 1.4 & $<7.5$ & $-0.16 \pm 0.03 \pm 0.07(34)$ & $-0.15 \pm 0.05 \pm 0.08$ \\
\hline Dolan24 & $0.05 \pm 0.03$ & 5350 & 4.3 & 1.4 & $<7.5$ & $0.01 \pm 0.02 \pm 0.06(54)$ & $0.1 \pm 0.1 \pm 0.08$ \\
\hline
\end{tabular}

that of the given ONC star. For each line, the quantity $r_{\text {line }}$ was calculated as $E W_{\mathrm{IC}} / E W_{\mathrm{ONC}}-1$. We did not find any trend of $r_{\text {line }}$ with wavelength for none of the stars, similarly to the results of Santos et al. (2008). Hence, we computed the average value of $r$ for each star using all lines. These final values are listed in Col. 2 of Tables 3 and 4. The two tables show that $r$ is consistent with zero for all stars with exception of the ONC members h278, for which we obtained $r=0.8 \pm 0.07$; due to this relatively high veiling, the star was discarded from the sample.

\subsection{Abundance analysis}

The analysis was performed using MOOG (Sneden 1973-2002 version) and a grid of 1-D model atmospheres from Kurucz (1993), by means of both EWs and spectral synthesis. Radiative and Stark broadening are treated in a standard way in MOOG; as for collisional broadening, we used the Unsöld approximation (1955) for all the lines. As discussed by Paulson et al. (2003) this choice should not greatly affect the differential analysis with respect to the Sun. We also mention that very strong lines $(E W \geq 150 \mathrm{~m} \AA)$ that are most affected by the treatment of damping have been excluded from our analysis.

\subsubsection{EW analysis and stellar parameters}

As mentioned earlier, the spectrum of the $\lambda$ Ori star was acquired using the CD3 and the analysis was done using the line list of Randich et al. (2006) who had used the same UVES set-up. For the ONC targets and the spectrum retrieved from the archive that were observed with the CD4, we instead built a new line list. Although the spectra cover the wavelength interval 6700 to $10000 \AA$, only the blue part up to $\sim 8000 \AA$ was usable, since the red part was contaminated by several telluric lines which could not be corrected, owing to the lack of spectra of earlytype stars. Fe I features to be used for the analysis in the interval 6700-8000 A were selected from different sources in the literature and subsequently checked for suitability (e.g. for blends) both on the solar spectrum observed with UVES and on the best quality sample spectra. The final list contains $40 \mathrm{Fe}$ I lines for the warm star HD 294297 and 26 lines for the cool members of the ONC (see Table 5); 24 lines are in common. Not for all the cool stars, however, it was possible to measure all the 26 lines. Most log $g f$ values were taken from Clementini et al. (1999); for the few lines not included in that study, $\log g f$ were instead retrieved from the Vienna Astronomical Line Database $\left(V_{A L D}^{3}\right)$. We performed the analysis of the solar spectrum obtained with UVES at the same resolution as that of our sample spectra using both the line list with 40 lines and that with 26 lines. Adopting as solar parameters $T_{\text {eff } \odot}=5770 \mathrm{~K}, \log g_{\odot}=4.44$ and $\xi_{\odot}=1.1 \mathrm{~km} \mathrm{~s}^{-1}$ (see Randich et al. 2006), with both line lists we obtained $\log n(\mathrm{Fe})=7.50 \pm 0.03$, a value very close to $\log n(\mathrm{Fe})_{\odot}=7.52$ (Anders \& Grevesse 1989). Individual $\log n(\mathrm{Fe})$ values for the Sun are listed in Table 5. $[\mathrm{Fe} / \mathrm{H}]$ for the sample stars with CD4 spectra was derived differentially with respect to our own determination. For Dolan 24, analyzed using the line list of Randich et al., a value $\log n(\mathrm{Fe})_{\odot}=7.52$ was instead adopted.

$E W$ s were measured using the package SPECTRE and a Gaussian fitting procedure. Although the spectra had been

${ }^{3}$ http://ams.astro.univie.ac.at/cgi-bin/vald/ 
Table 5. Line list of $42 \mathrm{Fe}$ I lines used for the analysis of the ONC stars along with excitation potential $(\chi), \log g f$ values, equivalent widths measured on the solar spectrum acquired with UVES and corresponding Fe abundances.

\begin{tabular}{|c|c|c|c|c|}
\hline $\begin{array}{l}\lambda \\
(\AA) \\
\end{array}$ & $\begin{array}{c}\chi \\
(\mathrm{EV}) \\
\end{array}$ & $\overline{l \log g f}$ & $\begin{array}{l}E W_{\odot} \\
(\mathrm{m} \AA)\end{array}$ & $\overline{\log n(\mathrm{Fe})_{\odot}}$ \\
\hline 6703.576 & 2.760 & -3.100 & 37 & 7.53 \\
\hline $6713.745^{\mathrm{w}}$ & 4.790 & -1.410 & 23 & 7.50 \\
\hline $6726.673^{w}$ & 4.610 & -1.050 & 48 & 7.50 \\
\hline 6750.164 & 2.420 & -2.655 & 75 & 7.51 \\
\hline 6786.860 & 4.190 & -1.900 & 26 & 7.49 \\
\hline 6806.856 & 2.730 & -3.140 & 36 & 7.51 \\
\hline 6810.267 & 4.610 & -1.000 & 50 & 7.49 \\
\hline $6820.374^{\mathrm{w}}$ & 4.640 & -1.160 & 41 & 7.51 \\
\hline 6839.835 & 2.560 & -3.450 & 29 & 7.50 \\
\hline $6843.655^{\mathrm{w}}$ & 4.550 & -0.860 & 63 & 7.53 \\
\hline 6858.155 & 4.610 & -0.950 & 52 & 7.48 \\
\hline $6862.496^{\mathrm{w}}$ & 4.560 & -1.430 & 33 & 7.53 \\
\hline 6945.200 & 2.420 & -2.460 & 81 & 7.41 \\
\hline 6951.250 & 4.560 & -1.050 & 52 & 7.52 \\
\hline $6978.860^{\mathrm{w}}$ & 2.480 & -2.490 & 84 & 7.56 \\
\hline 6988.530 & 2.400 & -3.420 & 39 & 7.51 \\
\hline 7022.960 & 4.190 & -1.110 & 67 & 7.51 \\
\hline $7024.070^{w}$ & 4.070 & -1.940 & 30 & 7.49 \\
\hline $7038.230^{\mathrm{w}}$ & 4.220 & -1.130 & 62 & 7.47 \\
\hline $7083.400^{\mathrm{w}}$ & 4.910 & -1.260 & 24 & 7.48 \\
\hline $7142.520^{\mathrm{w}}$ & 4.950 & -0.930 & 39 & 7.51 \\
\hline 7219.690 & 4.070 & -1.570 & 50 & 7.52 \\
\hline 7221.210 & 4.560 & -1.220 & 42 & 7.49 \\
\hline $7228.700^{c}$ & 2.760 & -3.270 & 27 & 7.45 \\
\hline 7284.840 & 4.140 & -1.630 & 43 & 7.51 \\
\hline 7306.570 & 4.180 & -1.550 & 43 & 7.47 \\
\hline $7401.690^{\mathrm{w}}$ & 4.190 & -1.600 & 44 & 7.54 \\
\hline 7418.670 & 4.140 & -1.440 & 51 & 7.47 \\
\hline $7421.560^{\mathrm{w}}$ & 4.640 & -1.690 & 20 & 7.52 \\
\hline 7447.400 & 4.950 & -0.950 & 35 & 7.44 \\
\hline $7461.530^{c}$ & 2.560 & -3.450 & 30 & 7.49 \\
\hline 7491.660 & 4.300 & -1.010 & 70 & 7.54 \\
\hline 7507.270 & 4.410 & -1.030 & 62 & 7.52 \\
\hline 7531.150 & 4.370 & -0.640 & 90 & 7.58 \\
\hline $7547.900^{w}$ & 5.100 & -1.110 & 25 & 7.51 \\
\hline 7568.910 & 4.280 & -0.900 & 76 & 7.52 \\
\hline 7583.800 & 3.020 & -1.930 & 85 & 7.49 \\
\hline 7710.363 & 4.220 & -1.112 & 66 & 7.48 \\
\hline $7751.110^{\mathrm{w}}$ & 4.990 & -0.740 & 48 & 7.50 \\
\hline $7807.910^{\mathrm{w}}$ & 4.990 & -0.510 & 61 & 7.50 \\
\hline 7912.870 & 0.860 & -4.850 & 49 & 7.50 \\
\hline $7959.150^{\mathrm{w}}$ & 5.030 & -1.180 & 24 & 7.47 \\
\hline
\end{tabular}

Lines labeled with a " $w$ " or "c" have been used for the warm star HD 294297 or for the cool ONC stars only.

previously normalized, local continuum was inspected and, if needed, adjusted at each $E W$ measurement. Initial stellar parameters were estimated as follows: $i$ ). effective temperatures for the ONC stars were retrieved from Hillenbrand (1997) who, in turn, had derived them from spectral-types using the scale of Kenyon \& Hartmann (1995). $T_{\text {eff }}$ of HD 294297 was taken from Cunha et al. (1998), while $T_{\text {eff }}$ for the $\lambda$ Ori star, for which the spectraltype is not available, was derived from the $R-I$ color and again the scale of Kenyon \& Hartmann. A reddening $E(R-I)=0.07$ was adopted (see discussion in Dolan \& Mathieu 1999); ii). an initial microturbulence $\xi=1.5 \mathrm{~km} \mathrm{~s}^{-1}$ was adopted for all stars; iii). finally, surface gravities for the ONC stars and Dolan 24 were estimated from the relation between $M, L$, and $T_{\text {eff }}(\log g=$ $\left.4.44+\log \left(M / M_{\odot}\right)-\log \left(L / L_{\odot}\right)+4 \log T_{\text {eff }}-15.0447\right)$. For the ONC stars masses and luminosities were taken from
Getman et al. (2005); for the star Dolan 24 in $\lambda$ Ori we derived the bolometric luminosity from the $I_{\mathrm{C}}$ magnitude, applying the bolometric correction by Bessell (1991) and adopting a distance to the cluster equal to $400 \mathrm{pc}$. Mass was then estimated using the PMS evolutionary tracks by Baraffe et al. (1998) with $\alpha=1$. For HD 294297 we adopted the log $g$ value of Cunha et al. (1998).

As usually done, final temperatures were derived by removing the trends between abundance values $(\log n(\mathrm{Fe}))$ and excitation potential $(\chi)$ in MOOG. Similarly, final $\xi$ values were derived by removing the trend between $\log n(\mathrm{Fe})$ and measured $E W$ s. In both cases a $2 \sigma$ clipping was applied to the initial line list before removing the trends. On the other hand, because of the lack of suitable Fe II lines, $\log g$ values could not be optimized for any of the stars and initial values were adopted.

Final stellar parameters are listed in Tables 3 and 4. We first note that for HD 294297 we retrieve the same $T_{\text {eff }}$ as Cunha et al. (1998). Then, the comparison of Tables 1 and 2 with Tables 3 and 4 shows that the maximum difference between final and initial effective temperatures is on the order of $100 \mathrm{~K}$, with the exception of star h673 $\left(\Delta T_{\text {eff }} \sim 300 \mathrm{~K}\right)$ and Dolan24 $\left(\Delta T_{\text {eff }}=270 \mathrm{~K}\right)$. All final temperatures are warmer than initial ones. Tables 3 and 4 also indicate that the final microturbulence values are in the range $\sim 1.5-1.7 \mathrm{~km} \mathrm{~s}^{-1}$, only slightly larger than those normally found for main sequence dwarfs of similar temperature. For the PMS stars in their sample, and in particular for the three ONC members, Santos et al. (2008) instead found higher values (up to $\xi=2.5 \mathrm{~km} \mathrm{~s}^{-1}$ ) and a larger star-to-star scatter. They suggested that the discrepancy between their microturbulence values and those of main sequence stars might be due to the effect of strong magnetic fields that characterize very young stars. Our results do not seem to support this hypothesis, since the microturbulence values for our sample stars are only slightly higher than that of the main sequence member of IC 2391 (see Sect. 4.4 below). Note that use of higher values of the microturbulence would lead to lower $[\mathrm{Fe} / \mathrm{H}]$ values.

The iron abundance for each line was obtained based on measured $E W \mathrm{~s}$ and adopted stellar parameters. Final abundances for each star were then determined as the mean abundance from the different lines.

\subsubsection{Spectral synthesis}

The spectral synthesis for the Fe analysis was performed in a $20 \AA$ wavelength range around the Li I feature at $6707.78 \AA$, from $6695 \AA$ to $6715 \AA$. As in the case of the $E W \mathrm{~s}$, we first carried out an analysis of the solar spectra acquired with UVES and Giraffe. When necessary, we modified $\log g f$ values to get a good fit of the solar spectrum with the standard solar abundances of Anders \& Grevesse (1989).

The synthesis was carried out for all but one star observed with UVES, in order to cross-check $[\mathrm{Fe} / \mathrm{H}]$ from $E W \mathrm{~s}$; spectral synthesis was also performed for the two ONC Giraffe spectra, whose lower resolution and reduced spectral range did not allow us to carry out the $E W$ analysis. Also note that, due to the relatively high rotation, $[\mathrm{Fe} / \mathrm{H}]$ for $\mathrm{h} 907$ in $\mathrm{ONC}$ could be measured only from the synthesis analysis. Instead, for the star h223a in the ONC we could not apply the synthesis method because part of the bluest order, including the wavelength range from 6695 to $6740 \AA$, fell out of the CCD.

For stars observed with UVES, the synthesis was carried out adopting the final stellar parameters determined with the $E W$ analysis; on the other hand, stellar parameters could not be optimized for stars observed with Giraffe and for h907, for which we 

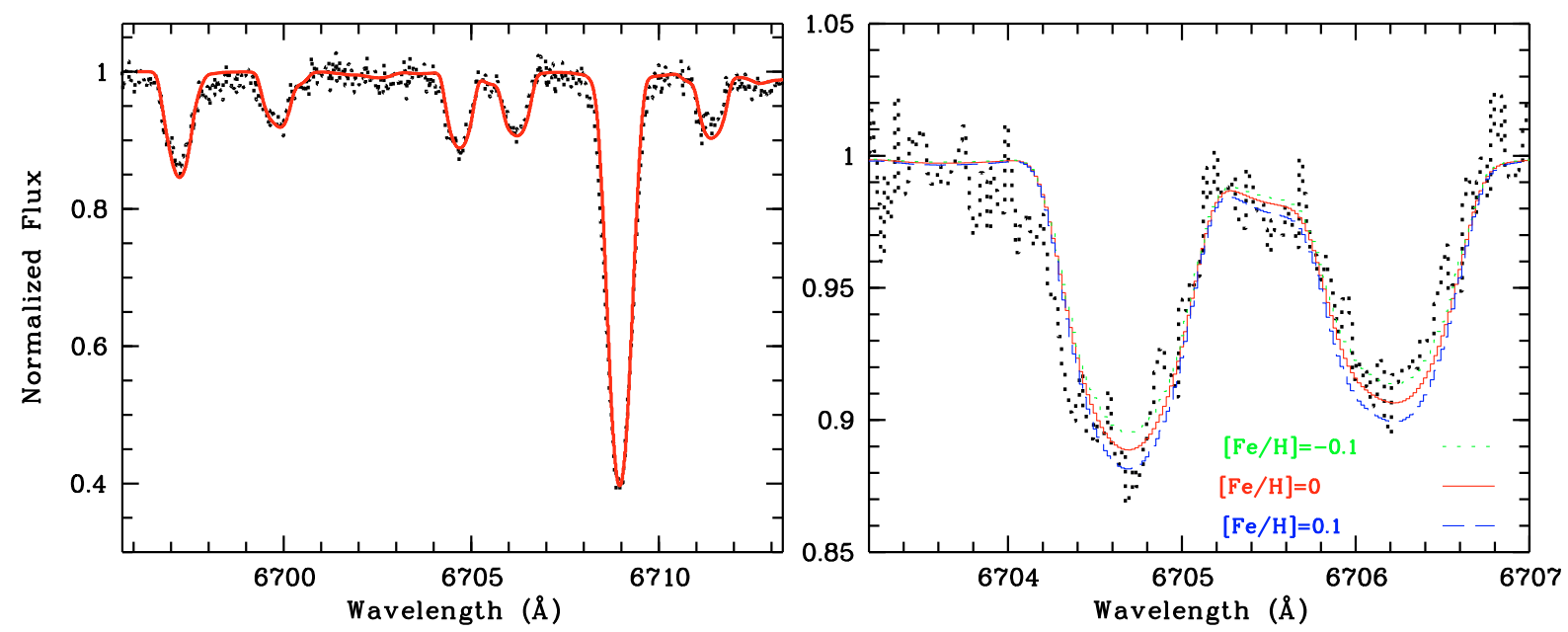

Fig. 3. Left panel: observed spectrum (dashed line) and best-fit synthetic spectrum (solid line, $[\mathrm{Fe} / \mathrm{H}]=0)$ for the star h673. Right panel: zoom on a restricted spectral region. Spectral synthesis with three different values of the metallicity are shown: $[\mathrm{Fe} / \mathrm{H}]=0$ (solid, red), 0.1 (dashed, blue), -0.1 (dotted, green).
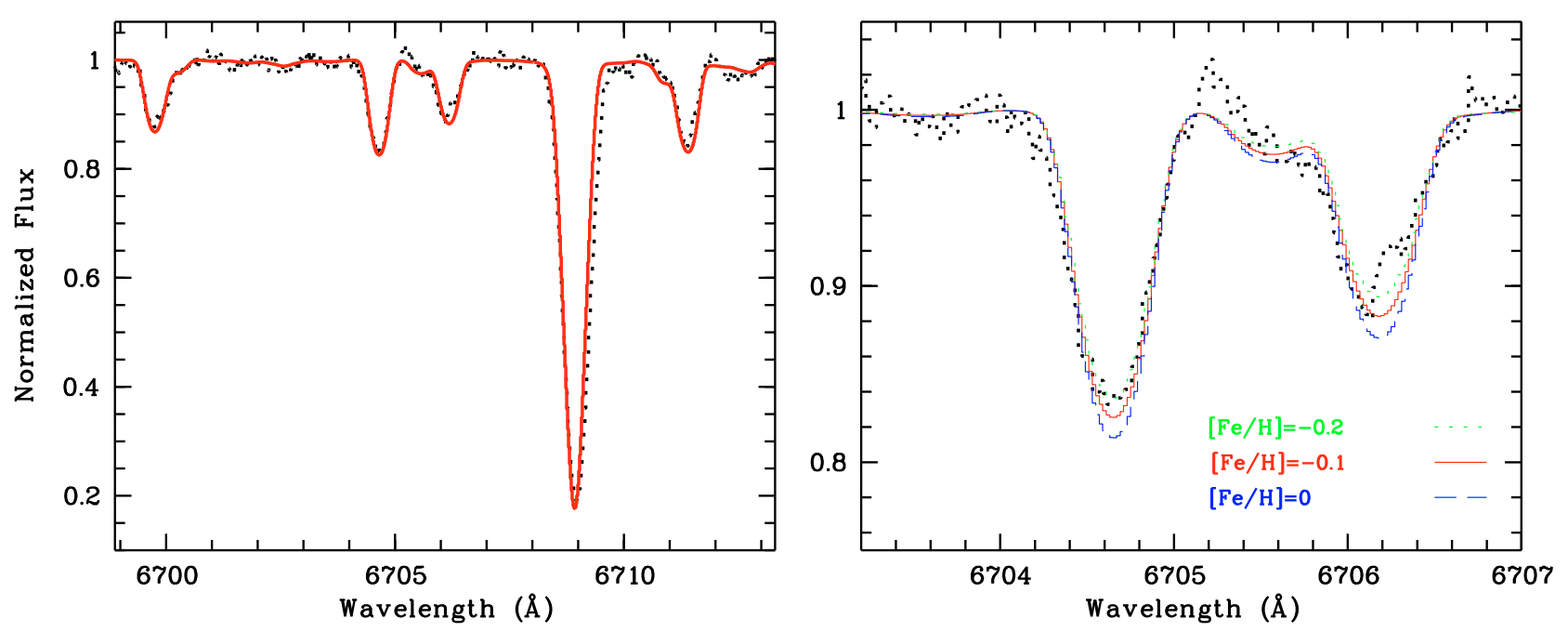

Fig. 4. Same as Fig. 3, but the observed and synthetic spectra of star h683 are shown. Note that the $[\mathrm{Fe} / \mathrm{H}]$ from $E W$ s for this star is slightly larger than that from the synthesis; the figure shows that the synthetic spectrum with $[\mathrm{Fe} / \mathrm{H}]=-0.1$ better fits the observed spectrum.

did not carry out the $E W$ analysis. Therefore, for these stars we adopted initial $T_{\text {eff }}$ values and a microturbulence $\xi=1.6 \mathrm{~km} \mathrm{~s}^{-1}$, which is typical of our UVES sample. Also, for each UVES spectrum, we first computed a synthetic spectrum with the metallicity determined through the $E W$ analysis; then, if necessary, we changed the metallicity until the best fit of the observed spectrum was obtained. For the analysis of the Giraffe spectra and that of h907 we started with a solar metallicity. Examples of spectral synthesis in the 6695-6715 A region for UVES and Giraffe spectra are shown in Figs. 3 (star h673), 4 (star h683), and 5 (star h664), respectively. In all figures, both the whole spectral region and a zoom around two Fe I lines are displayed. Those two lines have different excitation potentials and thus their strength has a different dependence on effective temperature. A large error in the determination of the latter would thus be identified in the comparison of synthetic and observed spectra. We also note that the red side of the Li I $6707.8 \AA$ feature is not well fitted in the spectra of stars h683 and h664 (Figs. 4 and 5). Although a detailed investigation of this effect is outside the purposes of this paper, we suggest that it might be due to the contribution of ${ }^{6} \mathrm{Li}$ to the lithium feature. ${ }^{6} \mathrm{Li}$ is indeed not included in our current line list. We finally notice that, as a by-product, spectral synthesis also allowed us to estimate projected rotational velocities $(v \sin i)$ for the sample stars. The latter are listed in Col. 6 of Tables 3 and 4.

\subsection{Errors}

Three sources of random errors are present in the $E W$ analysis: (i) $\sigma_{1}$, the standard deviation around the mean due to differences in abundances that come from each line; $\sigma_{1}$ should be representative of errors in $E W \mathrm{~s}$ and $\log g f$ values; (ii) $\sigma_{2}$ : errors due to uncertainties on the stellar parameters. We found that for all the stars variations in $T_{\text {eff }}$ greater than $100 \mathrm{~K}$ would produce significant trends in $\log n(\mathrm{Fe})$ versus excitation potential, while variations greater than $0.15 \mathrm{~km} \mathrm{~s}^{-1}$ in $\xi$ would introduce a trend between $\log n(\mathrm{Fe})$ and measured $E W \mathrm{~s}$; we thus assumed $100 \mathrm{~K}$ and $0.15 \mathrm{~km} \mathrm{~s}^{-1}$ as typical uncertainties in $T_{\text {eff }}$ and $\xi$. As to gravity, we adopted a typical uncertainty of 0.25 dex. Resulting errors in $[\mathrm{Fe} / \mathrm{H}]$ were then estimated by varying one parameter at the time and quadratically adding the resulting errors. We found that a change in $T_{\text {eff }}$ of $\pm 100 \mathrm{~K}$ results in a change of Fe abundance 



Fig. 5. Same as Figs. 3 and 4, but the observed and synthetic spectra of star h664 observed with Giraffe are shown. Note that Giraffe wavelength scale is in nm rather than in $\AA$.

from \pm 0.01 to \pm 0.04 dex; a change in $\xi$ of $\pm 0.15 \mathrm{~km} \mathrm{~s}^{-1}$ causes a change of $\pm 0.03 \mathrm{dex}$, and a variation of $0.25 \mathrm{dex}$ in $\log \mathrm{g}$ results in a change from \pm 0.04 to \pm 0.07 dex in abundance; (iii) $\sigma_{3}$ : errors due to uncertainties on veiling determination. As shown in Tables 3 and 4, the last are very small and lead to an error in the final $[\mathrm{Fe} / \mathrm{H}]$ on the order of $0.01-0.02$ dex, much below the other two errors. Also note that those small errors on veiling do not introduce any effect on the determination of stellar parameters.

The abundance uncertainties are related to the best fit determination $(\sigma 1)$ and to the uncertainties in stellar parameters $(\sigma 2)$, also in the case of spectral synthesis analysis. Assuming the same uncertainties in stellar parameters derived from the $E W$ analysis, we found that a change in $T_{\text {eff }}$ of $\pm 100 \mathrm{~K}$ results in a difference in $\mathrm{Fe}$ abundance from \pm 0.05 to $\pm 0.1 \mathrm{dex}$, while a change of $\xi$ of $\pm 0.15 \mathrm{~km} \mathrm{~s}^{-1}$ causes a variation from \pm 0.03 to \pm 0.05 dex in abundance values. The effect of gravity is instead negligible (below $0.02 \mathrm{dex}$ ).

Systematic errors that could affect the analysis of cool stars were estimated by analyzing with the same method a star with similar parameters as our sample stars and known metallicity. We chose a member of the open cluster IC 2391 (VXR76A) whose metallicity has been estimated with an independent method (different line list and code) by Randich et al. (2001 $[\mathrm{Fe} / \mathrm{H}]=0.0 \pm 0.09 \pm 0.09)$. For the analysis of VXR76A we used the same spectrum employed by Randich et al., characterized by a spectral resolution $R=43800$, very similar to that of the UVES spectra. We derived the metallicity of VXR76A using both $E W \mathrm{~s}$ and the two line lists and spectral synthesis. The results are listed in the last raw of Table 3. In all cases the agreement with the previous determination is excellent: namely, $[\mathrm{Fe} / \mathrm{H}]=0.0 \pm 0.06 \pm 0.08$ from the $E W \mathrm{~s}$ and the CD3 line list, $[\mathrm{Fe} / \mathrm{H}]=-0.01 \pm 0.08 \pm 0.08$ from the $E W \mathrm{~s}$ and CD4 line list, and $[\mathrm{Fe} / \mathrm{H}]=0 \pm 0.1 \pm 0.1$ from the spectral synthesis. Furthermore, in a more recent analysis of a wider sample of stars and better quality spectra of IC 2391, D'Orazi \& Randich (2009) not only confirmed a solar metallicity for this cluster, but also found that VXR76A shares the same metallicity as other, warmer members in IC 2391. This suggests that our analysis should not be affected by major systematic errors and that no major offset should be present between the metallicity of cool and warmer stars. As for Dolan 24, the method/line list was already tested by Randich et al. (2006) on M 67 members.

\section{Results and discussion}

\subsection{The metallicity of the ONC}

The results of our analysis are summarized in Tables 3 and 4, where we give the veiling value $r$ (Col. 2), the adopted $T_{\text {eff }}, \log g$, and $\xi$ values (Cols. 3-5), vsin $i$ (Col. 6), [Fe/H] derived from $E W \mathrm{~s}$ analysis (Col. 7), along with the number of lines employed for the analysis. $[\mathrm{Fe} / \mathrm{H}]$ values from spectral synthesis are listed in Col. 8.

First, the two tables show that, in general, $[\mathrm{Fe} / \mathrm{H}]$ values from $E W$ s and spectral synthesis well agree within the uncertainties. We derived the mean ONC metallicity considering the more secure $[\mathrm{Fe} / \mathrm{H}]$ from $E W \mathrm{~s}$ for stars with both determinations; $[\mathrm{Fe} / \mathrm{H}]$ from synthesis was instead considered in the three cases for which the $E W$ analysis could not be performed. We find $[\mathrm{Fe} / \mathrm{H}]_{\mathrm{av}}=-0.01 \pm 0.04$, i.e., the ONC has a solar metallicity. Including only stars with $[\mathrm{Fe} / \mathrm{H}]$ from $E W \mathrm{~s}$, we would obtain $[\mathrm{Fe} / \mathrm{H}]_{\mathrm{av}}=-0.02 \pm 0.05$.

As mentioned, Santos et al. (2008), based on three stars, derived a significantly lower average metallicity for the ONC $([\mathrm{Fe} / \mathrm{H}]=-0.13 \pm 0.06)$, although the $[\mathrm{Fe} / \mathrm{H}]$ of the most metal-rich star in their sample is comparable to the most metalpoor one in our own sample. The discrepancy is likely due to the higher microturbulence values obtained in that study (see Sect. 4.3.1). As in our case, Santos et al. have used FLAMES with the fiber link to UVES and their spectra are of similar quality. For the analysis they have employed the same code and model atmospheres; however, they adopted a different line list and $\sigma$-clipping criterion. We suggest that this affects the microturbulence determination and thus the final $[\mathrm{Fe} / \mathrm{H}]$. We stress again that the comparison with VXR76A provides confidence in our results. As discussed in Sect. 1, the metallicity of the ONC has so far been poorly constrained, with some studies suggesting an underabundance with respect to the Sun and others finding solar or even over-solar $[\mathrm{Fe} / \mathrm{H}]$ values. Iron has been determined in eight low-mass stars (1 from Cunha et al. 1998; four from Padgett 1996; and three from Santos et al. 2008) and one B-type star (Cunha \& Lambert 1994), but the $[\mathrm{Fe} / \mathrm{H}]$ values are not homogeneous, since they were measured with different techniques. Our study, not only almost doubles the number of ONC lowmass members with a metallicity measurement, but our $[\mathrm{Fe} / \mathrm{H}]$ 


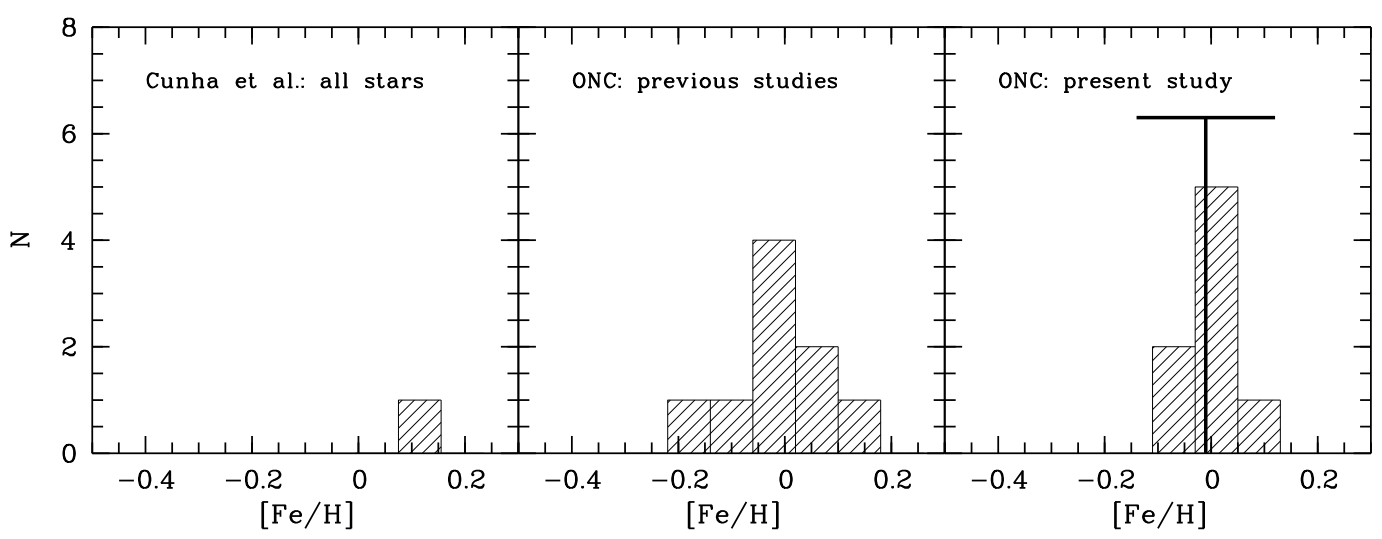

Fig. 6. Left panel: $[\mathrm{Fe} / \mathrm{H}]$ distribution across all the four Orion subgroups from Cunha et al. $(1994,1998)$; middle panel: $[\mathrm{Fe} / \mathrm{H}]$ distribution of the ONC only, obtained considering four different studies (Cunha \& Lambert 1994; Cunha et al. 1998; Padgett 1996; Santos et al. 2008); right panel: $[\mathrm{Fe} / \mathrm{H}]$ distribution of the $\mathrm{ONC}$ from our study. We also indicate our average for the $\mathrm{ONC}$ (vertical line) and the typical random error on $[\mathrm{Fe} / \mathrm{H}]$ of individual stars.

are homogeneous, thus allowing us to secure the average metallicity of the ONC on more solid grounds. The left panel of Fig. 6 shows the distribution of $[\mathrm{Fe} / \mathrm{H}]$ for the sample of Cunha et al. (1994, 1998) including both early- and late-type stars in all the four subgroups; in the middle panel we plot the $[\mathrm{Fe} / \mathrm{H}]$ distribution of ONC only, considering all previous determinations from the literature; finally, the distribution of our own measurements for ONC members is displayed in the right-hand panel, along with our average and the typical error on $[\mathrm{Fe} / \mathrm{H}]$ of individual stars. The figure shows that the distribution is very broad when including other subgroups besides the ONC, possibly implying a group-to-group difference; the distribution becomes narrower when considering the ONC only and measurements from different groups, but still spans $\sim 0.4$ dex in $[\mathrm{Fe} / \mathrm{H}]$. The distribution of our own $[\mathrm{Fe} / \mathrm{H}]$ values is instead much narrower and the small dispersion still present is completely due to measurement uncertainties.

As to OB1b, our [Fe/H] determination for star HD 294297 is in excellent agreement with the value of Cunha et al. (1998 $-[\mathrm{Fe} / \mathrm{H}]=-0.19)$. For the same star González-Hernandéz et al. (2008) derived $[\mathrm{Fe} / \mathrm{H}]=-0.09 \pm 0.1$ (star SO000041 in their list), also in good agreement. All the three measurements indicate a sub-solar metallicity, much below our average for the ONC. In our study the $[\mathrm{Fe} / \mathrm{H}]$ values for this star and the ONC are on the same scale; we mention in passing that, by using for the analysis of HD 294297 the same subset of lines used for the ONC, we would still obtain for this star the same $[\mathrm{Fe} / \mathrm{H}]$. This suggests that the difference between the ONC stars and HD 294297 is real and that the OB1b group might be more metal poor than the ONC. We further develop this point in the next section.

Finally, the metallicity of $\lambda$ Ori is likely solar; whereas this represents the first measurement of the metallicity in this cluster, it is based on one star only and, obviously, additional measurements are needed.

\subsection{The iron distribution in Orion}

In Fig. 7 we plot the spatial distribution of stars in the different Orion subgroups with an available metallicity determination; stars in three metallicity bins are indicated with symbols of different size. For the ONC we show our average measurement.

The figure indicates that the three stars belonging to the OB1b subgroup all have $[\mathrm{Fe} / \mathrm{H}]$ below the average of the ONC and, possibly, of the 1c subgroup, whose metallicity is characterized by a larger uncertainty and dispersion. Two of the stars in the OB1b sample are B-type stars from Cunha \& Lambert (1994), while the third one is HD 294297; for this star we adopt our own $[\mathrm{Fe} / \mathrm{H}]$ determination. The resulting average metallicity for the $1 \mathrm{~b}$ subgroup is $[\mathrm{Fe} / \mathrm{H}]=-0.10 \pm 0.05,2 \sigma$ below our determination for the ONC. The difference is not big and based on very few objects only, two of which are early-type stars. Nevertheless, it suggests that the Orion region may not be characterized by a single value of $[\mathrm{Fe} / \mathrm{H}]$. As well known, $\mathrm{Fe}$ is mostly produced by type-I Supernovae (SNe), whose lifetimes are much greater than the age of the Orion association $\left(\sim 10^{7}\right.$ years). On the other hand, massive, short-lived stars exploding as type-II SNe might pollute a molecular cloud close to OB associations. Although SNe II are mainly producer of $\alpha$ elements like $\mathrm{O}, \mathrm{Mg}$, and $\mathrm{Si}$, a small amount of $\mathrm{Fe}$ is also produced. As mentioned in Sect. 2, Cunha \& Lambert (1992) and Cunha et al. (1998) reported evidence of star-to-star variations in $\mathrm{O}$ and $\mathrm{Si}$ abundances within Orion, with a few $\mathrm{O} / \mathrm{Si}$-enhanced stars more centrally located in the Trapezium region than the widespread low $\mathrm{O} / \mathrm{Si}$ ones; they suggested a simple scenario of supernova self-enrichment across the Orion association over the past 10 Myr. With the caveat that the Fe yields from SNe II are highly uncertain, they estimated that the enhancement in oxygen of $\sim 0.3$ dex would correspond to an enhancement in $\mathrm{Fe}$ of $\sim 0.06$ dex. Whereas in their study they did not find such a variation, the $\sim 0.1$ dex difference in iron that we measure between the ONC and $1 \mathrm{~b}$ subgroup is consistent with this the scenario. The OB1a subgroup might also be metal-poor; three of the four members (all from Cunha \& Lambert 1994) have $[\mathrm{Fe} / \mathrm{H}]$ more than $1 \sigma$ below the ONC, while one star is overabundant with respect to the Sun and the ONC.

Finally, Fig. 7 shows that a dispersion in metallicity is present in OB1c. We suggest that the dispersion is likely due to both systematic differences between different studies (i.e., Cunha et al. versus Padgett) and to large uncertainties within a given study, as we have shown to be the case for the ONC.

\subsection{The lack of metal-rich stars}

One of the goals of our project is the search for metal-rich young low-mass stars. The first results presented here indicate that the ONC has a solar metallicity; the dispersion we find within the cluster is consistent with measurement errors and none of 


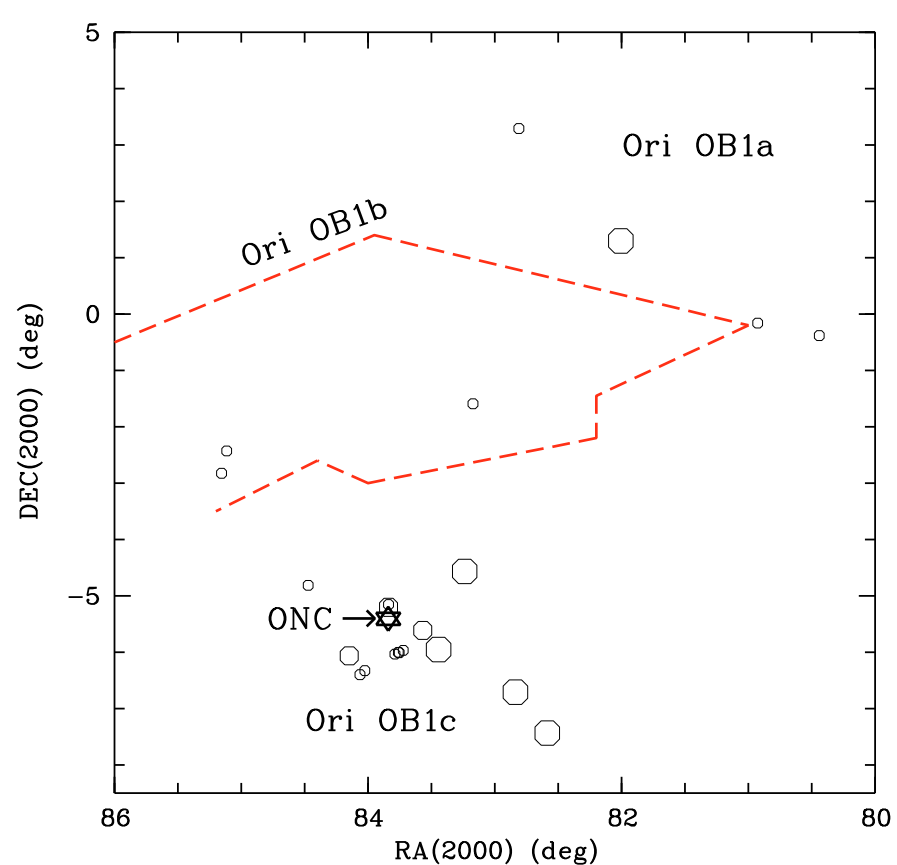

Fig. 7. Spatial distribution of members of the Orion association with $[\mathrm{Fe} / \mathrm{H}]$ measurements. Open symbols indicate members of the OB1a, $1 \mathrm{~b}$, $1 \mathrm{c}$ subgroups, while our average for the ONC is represented by the star symbol. Symbols with different size denote three different metallicity bins: $[\mathrm{Fe} / \mathrm{H}] \leq-0.05,-0.05<[\mathrm{Fe} / \mathrm{H}] \leq 0.03$, and $[\mathrm{Fe} / \mathrm{H}]>0.03$, from the smallest to the largest bin. The central bin corresponds to the average metallicity of the $\mathrm{ONC} \pm 1 \sigma$. The dashed line outlines the boundaries of the Ori OB1b subgroup following Warren \& Hesser (1977).

the stars in our sample is metal-rich. Under the assumption that our analysis is not affected by major systematic errors, as the comparison with IC 2391 confirms, two opposite hypothesis can be suggested to explain the lack of such a population. The simplest and most straightforward one is that they do not exist and, as expected within a cluster, the ONC is characterized by a homogeneous composition of all its members. Alternatively, the $[\mathrm{Fe} / \mathrm{H}]$ distribution of the $\mathrm{ONC}$ is inhomogeneous and metal-rich members exist, but low number statistics prevented us from detecting them. To this respect, we recall that one star from Padgett (1996) has a metallicity significantly above solar $([\mathrm{Fe} / \mathrm{H}]=0.14 \pm 0.18)$ and above our mean for the ONC. The final answer on the possible presence of metal-rich stars can only be provided by a metallicity determination in a much wider sample of stars, including also a re-analysis of the possible metal-rich ONC member from the study of Padgett. We caveat however that a large metallicity dispersion within the ONC itself would be difficult to explain without assuming ad-hoc processes. To our knowledge, abundance patterns in all open clusters so far investigated are rather homogeneous and no metal-rich members of solar-metallicity clusters or, viceversa, solar-metallicity members of metal-rich clusters have been detected.

On more general grounds, the question arises on the existence of metal-rich young associations and SFRs. None has been detected so-far (see the discussion in Santos et al. 2008). In Fig. 8 we show the metallicity distribution of open clusters within $500 \mathrm{pc}$ from the Sun with an available spectroscopic $[\mathrm{Fe} / \mathrm{H}]$ determination. The 16 clusters included in the sample have ages in the interval $30 \mathrm{Myr}-2 \mathrm{Gyr}$; the metallicity for all but one has been derived from high-resolution spectra of lowmass stars and typical errors on the average $[\mathrm{Fe} / \mathrm{H}]$ values are in the range $0.02-0.1$ dex. The figure shows that the distribution



Fig. 8. Metallicity distribution of clusters within $500 \mathrm{pc}$ from the Sun with a secure measurement of the metallicity; the average $\pm 1 \sigma$ for the $\mathrm{ONC}$ is indicated as a vertical bar.

peaks at solar $[\mathrm{Fe} / \mathrm{H}]$, with a not negligible tail at high values; specifically, metallicities vary between $[\mathrm{Fe} / \mathrm{H}] \sim-0.15$ and 0.4 with an average value $[\mathrm{Fe} / \mathrm{H}]=0.02 \pm 0.1$. In other words, the majority of the clusters in the solar neighborhood share the same metallicity as the Sun and the ONC is not peculiar. On the other hand, metal-rich open clusters do exist in the solar circle, and their number, and thus probability of finding them, is in principle not negligible $(3 / 16$ or $19 \%$ of the clusters have $[\mathrm{Fe} / \mathrm{H}]>0.1)$. However, whereas a strict $[\mathrm{Fe} / \mathrm{H}]$ versus age relationship does not hold, the four clusters with $[\mathrm{Fe} / \mathrm{H}] \geq 0.05$ are all older than 200 Myr; furthermore, two of them (Hyades and Praesepe) belong to the Hyades super-cluster or stream (Eggen 1992; Famæy et al. 2007) and might have a peculiar origin. The majority of the clusters with close-to-solar metallicity are instead part of the Local Association (or Pleiades moving group) or of the IC 2391 supercluster, to which various young associations such as Scorpius-Centaurus are also associated. Finally, the open clusters IC 4665 and IC 2391, both with a solar iron content are likely members of the Gould Belt (Piskunov et al. 2006). In other words, both kinematic and age considerations suggest that it might not be easy to find a metal-rich young association in the solar neighborhood.

\section{Concluding remarks}

Using FLAMES/UVES and Giraffe spectra, we determined iron abundances in eight low-mass members of the ONC, one star in the $\lambda$ Ori cluster, and one member of the Orion OB1b subgroup. The average metallicity of the $\mathrm{ONC}$ is $[\mathrm{Fe} / \mathrm{H}]=-0.01 \pm 0.04$; the metallicity of Dolan 24 in $\lambda$ Ori is also solar, while the star belonging to the OB1b subgroup is significantly more metalpoor than the ONC. The $\sim 0.1$ dex difference in the Fe content of the ONC and the OB1b subgroup is consistent with the selfpollution scenario originally proposed by Cunha and collaborators $(1992,1994,1998)$ for the Orion region.

None of our sample stars nor any of the SFRs so far studied is metal-rich. At the same time, known metal-rich open clusters are all older than $200 \mathrm{Myr}$, and none is spatially or kinematically associated to young nearby associations and SFRs. This suggests that finding the metal-rich SFRs among the best studied ones in the solar neighborhood might not be easy or doomed to failure. We suggest that in order to find them, one should possibly look 
outside the Gould Belt and the Local Association. High-spectral resolution observations of low-mass stars in SFRs at distances larger than $500 \mathrm{pc}$ might indeed be feasible with current instrumentation.

Acknowledgements. It is a pleasure to thank N. Santos and C. Melo for useful discussions. We thank the referee, Luca Pasquini, for very useful comments. This work has made extensive use of the services of WEBDA, ADS, CDS and of the catalog by W. Dias (http://www.astro.iag.usp.br/ wilton/). We acknowledge partial support from PRIN-INAF "Stellar clusters: test for stellar formation and evolution" (PI: F. Palla).

\section{References}

Anders, E., \& Grevesse, N. 1989, Geochim. Cosmochim. Acta, 53, 197 Baraffe, I., Chabrier, G., Allard, F., \& Hauschildt, P. H. 1998, A\&A, 337, 403 Bessell, M. 1991, AJ, 101, 662

Blaauw, A. 1991, ASI Ser., 342, 125

Blecha, A., \& Simond, G. 2004, GIRAFFE BLDRS Software Reference Manual, ver. 1.12, Geneve: Obs. Geneve

Caballero, J. A. 2007, A\&A, 466, 917

Clementini, G., Gratton, R. G., Carretta, E., \& Sneden, C. 1999, MNRAS, 302, 22

Cunha, K., \& Lambert, D. L. 1992, ApJ, 399, 586

Cunha, K., \& Lambert, D. L. 1994, ApJ, 426, 170

Cunha, K., Smith, V. V., \& Lambert, D. L. 1995, ApJ, 452, 634

Cunha, K., Smith, V. V., \& Lambert, D. L. 1998, ApJ, 493, 195

Dolan, C. J., \& Mathieu, R. 1999, AJ, 118, 2409

Dekker, H. 2000, SPIE, 4008, 534

D’Orazi, V., \& Randich, S. 2009, A\&A, 501, 553

Ecuvillon, A., Israelian, G., Santos, N. C., Mayor, M., \& Gilli, G. 2006, A\&A, 449, 809

Eggen, O. 1992, AJ, 104, 1482

Esteban, C., Peimbert, M., Garcìa-Rojas, J., et al. 2004, MNRAS, 355, 229

Famæy, B., Pont, F., Luri, X., et al. 2007, A\&A, 461, 957

Fischer, D. A., \& Valenti, J. A. 2005, ApJ, 622, 1102

Getman, K. V., Flaccomio, E., Broos, P. S., et al. 2005, ApJS, 160, 319
Gilli, G., Israelian, G., Ecuvillon, A., Santos, N. C., \& Mayor, M. 2006, A\&A, 449,723

Gonzalez, G. 1997, MNRAS, 285, 403

Gonzalez, G., Lawas, C., Tyagi, S., \& Reddy, B. E. 2001, AJ, 121, 432

González Hernandéz, J. I., Caballero, J. A., Rebolo, R., et al. 2008, A\&A, 490, 1135

Herbst, W., Bailer-Jones, C. A. L., Mundt, R., Meisenheimer, K., \& Wackermann, R. 2002, A\&A, 396, 513

Hillenbrand, L. A. 1997, AJ, 113, 1733

Hillenbrand, L. A., Strom, S. E., Calvet, N., et al. 1998, AJ, 116, 1816

Kenyon, S. J., \& Hartmann, L. H. 1995, ApJS, 101, 117

Kurucz, R. L. 1993, ATLAS9 Stellar Atmosphere Programs. Kurucz CD-ROM

No. 13, Cambridge, Mass: Smithsonian Astrophysical Observatory

Osterbrock, D. E., Tran, H., \& Veilleux, S. 1992, ApJ, 389, 196

Padgett, D. L. 1996, ApJ, 471, 847

Palla, F., Randich, S., Flaccomio, E., \& Pallavicini, R. 2005, ApJ, 626, L49

Palla, F., Randich, S., Pavlenko, Ya. V., Flaccomio, E., \& Pallavicini, R. 2007, ApJ, 659, L41

Pasquini, L., Avila, G., Allaert, E., et al. 2000, SPIE Conf., 4008, 129, Munich, March

Pasquini, L., Döllinger, M. P., Weiss, A., et al. 2007, A\&A, 473, 979

Paulson, D. B., Sneden, C., \& Cochran, W. D. 2003, AJ, 125, 3185

Pinsonneault, M. H., DePoy, D. L., \& Coffee, M. 2001, ApJ, 556, L59

Piskunov, A. E., Kharchenko, N. V., Röser, S., et al. 2006, A\&A, 445, 545

Randich, S., Pallavicini, R., Meola, G., Stauffer, J. R., \& Balachandran, S. C. 2001, A\&A, 372, 862

Randich, S., Primas, F., Sestito, P., Pasquini, L., \& Pallavicini, R. 2006, A\&A, 450, 557

Reeves, H. 1978, A\&A, 19, 215

Sacco, G. G., Franciosini, E., Randich, S., \& Pallavicini, R. 2008, A\&A, 488, 167

Santos, N. C., Israelian, G., \& Mayor, M. 2001, A\&A, 373, 1019

Santos, N. C., Melo, C., James, D. J., et al. 2008, A\&A, 480, 889

Sicilia-Aguilar, A., Hartmann, L. W., \& Szentgyorgyi, A. H. 2005, AJ, 129, 363

Sìmon-Dìaz, S., Herrero, A., Esteban, C., \& Najarro, F. 2006, A\&A, 448, 351S

Sneden, C. 1973, ApJ, 184, 839

Stauffer, J. R., Hartmann, L. W., Prosser, C. F., et al. 1997, ApJ, 479, 776

Unsöld., A. 1955, Physik der Sternatmosphären (Berlin: Springer-Verlag)

Warren, W. H., \& Hesser, J. E. 1977, ApJS, 34, 115 